Document downloaded from:

http://hdl.handle.net/10251/48191

This paper must be cited as:

Perez Garnes, M.; Martínez Ramos, C.; Barcia, JA.; Escobar Ivirico, JL.; Gomez Pinedo, UA.; Vallés Lluch, A.; Monleón Pradas, M. (2013). One-dimensional migration of olfactory ensheathing cells on synthetic materials: Experimental and numerical characterization. Cell Biochemistry and Biophysics. 65:21-36. doi:10.1007/s12013-012-9399-1.

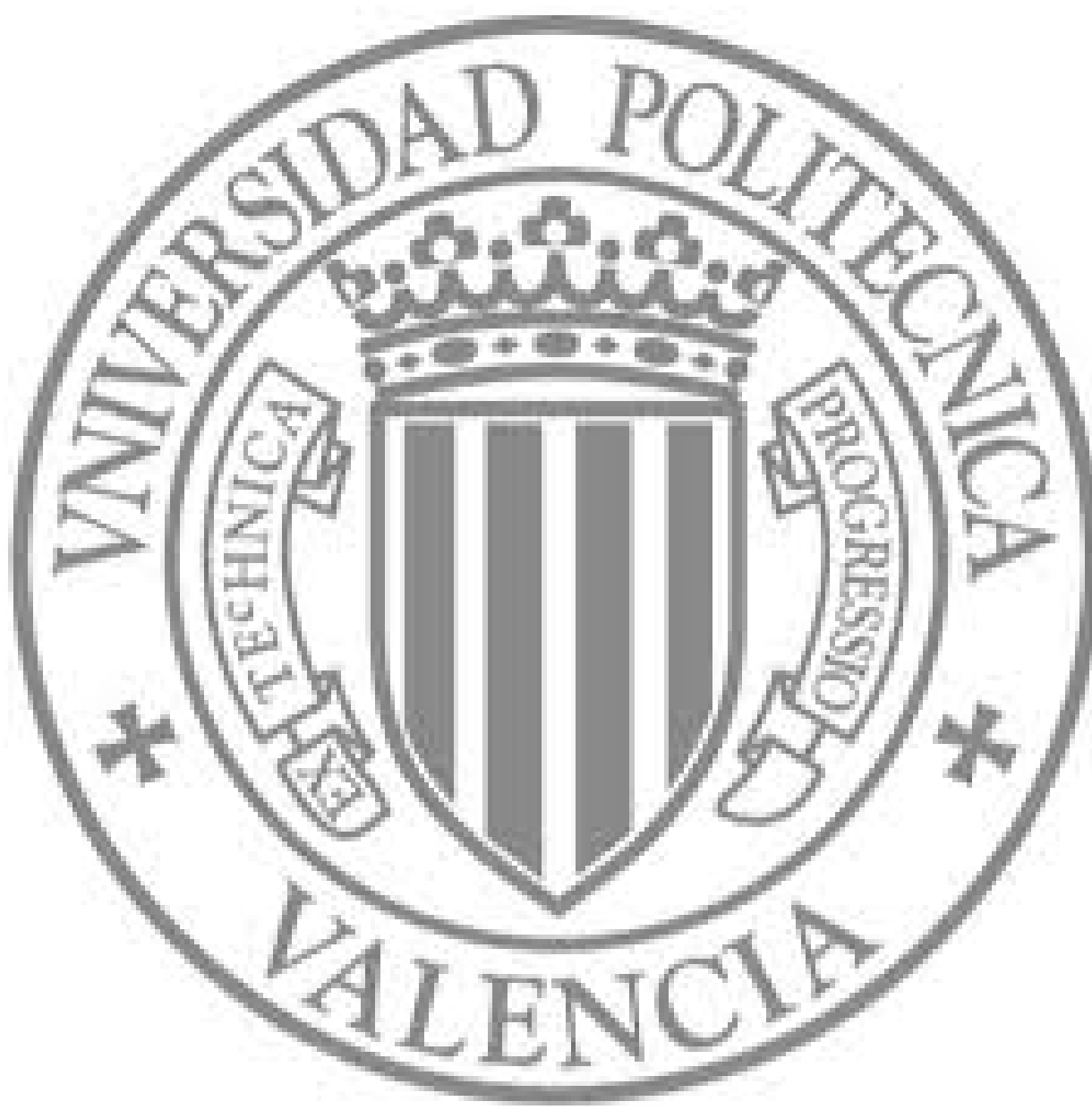

The final publication is available at

http://dx.doi.org/10.1007/s12013-012-9399-1

Copyright Humana Press 


\section{One-Dimensional Migration of Olfactory Ensheathing Cells on Synthetic Materials: Experimental and Numerical Characterization}

Manuel Pérez Garnes ${ }^{1 *}$, Cristina Martínez-Ramos ${ }^{1 *}$, Juan A. Barcia ${ }^{2}$, Jorge L. Escobar Ivirico $^{1}$, Ulises Gómez-Pinedo ${ }^{3}$, Ana Vallés Lluch ${ }^{1}$, Manuel Monleón Pradas**1,4

${ }^{1}$ Centro de Biomateriales e Ingeniería Tisular, Universitat Politècnica de València, Cno. de Vera s/n, 46022, Valencia, Spain

${ }^{2}$ Servicio de Neurocirugía, Hospital Clínico San Carlos, c/ Profesor Martín Lagos, s/n, 28040, Madrid, Spain

${ }^{3}$ Instituto de Neurociencias, Hospital Clínico San Carlos, c/ Profesor Martín Lagos, s/n, 28040 Madrid, Spain

${ }^{4}$ Networking Research Center on Bioengineering, Biomaterials and Nanomedicine (CIBER-BBN), Valencia, Spain

$\left(^{*}\right)$ Equal contribution

**Corresponding author.Tel.: +34963877277; E-mail: mmonleon@ter.upv.es 


\begin{abstract}
Olfactory ensheathing cells (OECs) are of great interest for regenerative purposes since they are believed to aid axonal growth. With the view set on strategies to achieve reconnection between neuronal structures it is of greatest importance to characterize the behaviour of these cells on long thread-like structures that may efficiently guide cell spread in a targeted way. Here rat OECs were studied on polycaprolactone long monofilaments, on long bars and on discs. Polycaprolactone turns out to be an excellent substrate for OECs. The cells cover long distances along the monofilaments, and colonize completely these structures. With the help of a one-dimensional analytical model a migration coefficient, a net proliferation rate constant, and the fraction of all cells which undergo migration were obtained. The separate effect of the three phenomena summarized by these parameters on the colonization patterns of the onedimensional paths was qualitatively discussed. Other features of interest were also determined, such as the speed of the advance front of colonization and the order of the kinetics of net cell proliferation. Characterizing migration by means of these quantities may be useful for comparing and predicting features of the colonization process (such as times, patterns, advance fronts, proportion of motile cells) of different cell-substrate combinations.
\end{abstract}

Keywords: olfactory ensheathing cells, polycaprolactone scaffolds, migration, diffusion, colonization. 


\section{Introduction}

Most neurological diseases implying damage of extensive brain structures lack nowadays a satisfying clinical treatment (Parkinson's disease, spinal cord injury, stroke, etc.). Some of the experimental therapies pursue the reconnection of axons and neurites, for which the guided growth of axons within or along filamentous structures might be useful (1-3). Olfactory ensheathing cells (OECs) or Schwann cells (SCs) are thought to help this process, by ensheathing and guiding the growing axons (4-9).

OECs reside in the olfactory neuroepithelium, and are considered permissive cells, may help axons to grow and populate both the olfactory nerve and the nerve fiber layer of the olfactory bulb (10). These cells are non-myelinating glial cells that ensheath olfactory axons $(11,12)$ and can penetrate the transition zone between the peripheral and central nervous systems. Several studies have shown that OECs present a great potential to guide axons in spinal cord injury (13-18) and in other neural diseases (19-22). In other studies, where peripheral nerves were used as bridges to link injured nerve tracts, the use of OECs showed effectiveness in reducing glial scar, modulate microglial reactivity and promote axonal growth (23-26).

The migration ability of OECs is essential for their role in neural regeneration (27), and this process is regulated by neurotrophic factors such as GDNF, NGF or BDNF (28-30). OECs may act as a pump of these neurotrophic factors, which provide the appropriate niche for axon projection and neuronal reconnection $(18,31)$. To facilitate axonal growth and guidance these cells produce extracellular matrix and cell adhesion molecules such as laminin (32), N-CAM (33) or L1 (34).

However, the direct injection of OECs in sites of lesion is limited by the complexity of the environmental interactions, including astrocytic reactivity, which may hinder or prevent the growth of axons. To facilitate this process of cell growth and guidance many researchers have started to develop biohybrids where OECs are seeded previously on different biomaterials (35-39). These kinds of structures have been produced from natural biomaterials such as alginate $(40)$, collagen $(41,42)$ and chitosan, synthetic polymers such as polyethylene glycol (43), polylactic acid (44) or poly-e-caprolactone $(39,43)$, and composite biomaterials (45). 
In this study we used poly- $\varepsilon$-caprolactone (PCL) because it is a biodegradable synthetic biomaterial widely tested in neural tissue engineering in vitro studies $(45,46)$ and in studies in vivo of neural damages such as traumatic brain injury $(47,48)$, spinal cord injury (49) and nerve regeneration $(50,51)$.

In the present work we cultured OECs on polycaprolactone (PCL) long monofilaments and bars, in order to characterize the colonization in time of these material structures by OECs seeded in one of the ends. This biohybrid of cells and filaments can be of use in trying to reconstruct axonal tracts and maybe other similar structures, since microfibers represent a constrained path for the cells to follow, and thus, so one hopes, they may be better targetted in their migration. Selected features of the process of cell migration and axon growth have been described by different mathematical models $(52,53)$. Individual cell motility is due to complex biochemical and biomechanical mechanisms of regulation of actin and myosin and external stimuli $(54,55)$ originating cell protrusion, adhesion and translocation $(56,57)$. Many mathematical models have been developed as discrete models studying biophysical mechanisms of individual cell motility (58-60). However, cells move in coordinated collective motion (61) and colonization cannot be deduced from individual movement only. Collective motion has been treated in macroscopic continuum models. Usually these models lead to a set of partial differential equations for the masses or concentrations of cells and chemicals (62-64). Normally the mass balance of cells is governed by a non-linear reaction-diffusion equation and the mass balance of chemicals by a diffusion equation. In vitro studies evidence that a large variety of cells show a travelling wave-like behaviour (65), and so several models are based on Fisher's equation (66), whose solutions determines a front of advance with a constant speed depending on the diffusivity and the net proliferation rate. The continuous models do not take into account small-scale processes and the random walk of cells, aspects which have been taken into account in hybrid models, which can combine certain biological aspects with the collective motion of the cells (67-69).

Here we studied the ability of OECs to migrate in vitro on PCL filaments, and the process was characterized analytically with the help of a reaction-diffusion model which gives numerical values of quantities such as the diffusion (or migration) coefficient of the cells, their advance front speed and their net proliferation rate. These parameters may facilitate the comparison of the colonization process of a given cell type on different materials for possible therapeutical applications. With such models, the 
equation parameters can be independently varied and thus the influence of the different phenomena involved in the colonization process, as described by those parameters (migration, proliferation), can be qualitatively assessed.

\section{Experimental Section}

\subsection{PCL bars and discs}

Polycaprolactone, PCL, (Polysciences, Inc., Warringtong, PA; MW 43000-50000 Da) was purchased in the form of pellets. $1 \mathrm{~mm}$-thick PCL films were obtained by compressing $10 \mathrm{~g}$ of pellets under a slight pressure $\left(2 \mathrm{~kg}\right.$ between two $10 \times 10 \mathrm{~cm}^{2}$ glass plates) in an oven for $3 \mathrm{~h}$ at $80^{\circ} \mathrm{C}$, then cooled in the oven until $40^{\circ} \mathrm{C}$ and afterwards in ambient until they reached room temperature and were unmolded. These PCL sheets were employed for the fabrication of bars and discs of $10 \mathrm{~mm}$ of diameter. The bars were cut having $30 \mathrm{~mm}$ length and $5 \mathrm{~mm}$ width, with a triangular end to facilitate later identification of the cell seeded end (see Figure 1A). At both ends of the bars, two smaller PCL discs ( $4 \mathrm{~mm}$ of diameter and $1 \mathrm{~mm}$-thick) were glued with a $25 \%(\mathrm{w} / \mathrm{w})$ $\mathrm{PCL} /$ dioxane solution and later compressed between two glass plates at $45^{\circ} \mathrm{C}$ for $1 \mathrm{~h}$, to provide a means to keep the bar raised over the well's bottom and to impede cell migration to the well. The discs and the bar constructs were sterilized with $70 \%$-ethanol (Scharlab, S.L. Barcelona, Spain) for $24 \mathrm{~h}$ and dried in vacuum before use.

\subsection{PCL filaments}

$80 \mu \mathrm{m}$-diameter PCL fibers were obtained by extrusion and drawing as follows: 200 micron-diameter filaments were extruded in a Haake Minilab II mixer (Thermo Fisher Scientific, Karlsruhe, Germany) starting from the same PCL pellets with a extrusion temperature of $80^{\circ} \mathrm{C}$, a torque between 9 and $12 \mathrm{~N} \cdot \mathrm{cm}$ and a rotation speed between 8 and $10 \mathrm{rpm}$. These fibers were then wound in groups of 16 and stretched in a stressstrain machine (Adamel Lhomargy, Division instruments S.A.) at ambient temperature at a rate of $20 \mathrm{~mm} / \mathrm{min}$ until the $95 \%$ of their breaking strength $(15 \mathrm{~N})$ to obtain PCL monofilaments of approximately $80 \mu \mathrm{m}$ of diameter. Five such filaments were mounted 
on PCL rings to obtain a manageable construct (Figure 1B) as follows. PCL rings were cut from the $1 \mathrm{~mm}$-thick PCL sheets with $10 \mathrm{~mm}$ of external and $8 \mathrm{~mm}$ of internal diameter; 5 stretched and aligned PCL monofilaments were glued on top of one of the ring's faces with a $25 \%(\mathrm{w} / \mathrm{w}) \mathrm{PCL} /$ dioxane solution. The resultant structures were sterilized with $70 \%$-ethanol for $24 \mathrm{~h}$ and dried in vacuum.

\subsection{Isolation and purification of cells}

OECs were isolated from the glomerular layer of olfactory bulbs. Briefly, Wistar rats (3 weeks old) were sacrificed by decapitation, with procedure approved by the institution's Ethical Committee. Olfactory bulbs were aseptically removed and treated with $0.25 \%$ trypsin, $0.1 \%$ collagenase A, and $0.1 \%$ DNase-I in $1 \mathrm{ml}$ of Hanks' balanced salt solution (Sigma, St. Louis, MO) with $\mathrm{Ca}^{2+}$ and $\mathrm{Mg}^{2+}$ at $37^{\circ} \mathrm{C}$ for $30 \mathrm{~min}$. Then, the enzymes were inactivated by addition of Dulbecco's minimum essential medium F-12 Ham (DMEM/F-12, Sigma) with 10\% inactivated fetal bovine serum (FBS, Invitrogen) and cells were recovered by centrifugation at $300 \mathrm{~g}$ for $8 \mathrm{~min}$.

Recovered cells were then resuspended in a mixture of (1.5:1) MEM 1X (Sigma, M0643) and DMEM/F-12 1X (Sigma, D8900) media, supplemented with 10\% inactivated FBS (Invitrogen, Cat 10108), $50 \mu \mathrm{l} / \mathrm{ml} \mathrm{L-glutamine} 200 \mathrm{mM}$ (Invitrogen, Cat 25030), $50 \mu \mathrm{l} / \mathrm{ml}$ penicillin-streptomycin 100X (Sigma, P0781), $0.5 \mu 1 / \mathrm{mL}$ gentamycin $50 \mathrm{mg} / \mathrm{ml}$ (Invitrogen, Cat 15750), $2 \mu \mathrm{l} / \mathrm{ml}$ MEM non-essential amino acid solution 100X (Sigma, M7145), $2 \mu \mathrm{l} / \mathrm{ml}$ MEM vitamin solution 100X (Sigma, M6895), and $1 \mu \mathrm{l} / \mathrm{ml}$ Forskolin $5 \mathrm{mg} / \mathrm{ml}$ (Sigma, F6886). Cells were then plated in a culture flask coated with poly-D-lysine $10 \mu \mathrm{g} / \mathrm{ml}$ (Sigma, P0899) and maintained at $37^{\circ} \mathrm{C}, 5 \% \mathrm{CO}_{2}$. The medium was changed every 2 days.

Cells reached confluence in monolayer after 8-10 days of culture; then, the OECs were purified by antibody based purification assay. The magnetic-activated cell separation (AutoMACS, Miltenyi Biotec, Germany) procedure was performed according to the manufacturer's protocol. The flask containing the unpurified cells was washed with Dulbecco's phosphate buffered saline (PBS) and incubated with $40 \mu 1 / \mathrm{cm}^{2}$ accutase (Invitrogen) for $5 \mathrm{~min}$ to detach the cells. Cells were collected in DMEM containing $10 \% \mathrm{FBS}$ and were centrifuged at $300 \mathrm{~g}$ for $5 \mathrm{~min}$. The pellet was rinsed-with PBS 
supplemented with $2 \mathrm{mM}$ EDTA (rinsing solution) and the cells were counted using a Neubauer counting chamber. For ensheathing cells selection, the cells were incubated with $5 \mu$ of anti-p75 LNGFr monoclonal antibodies (Chemicon, MAB 365) in $95 \mu 1$ Dulbecco's PBS, $2 \mathrm{mM}$ EDTA, 0.5\% BSA (Running Buffer) for $10 \mathrm{~min}$ at room temperature. After incubation time, $5 \mathrm{ml}$ of rinsing solution was added and the cells were newly centrifuged ( $300 \mathrm{~g}$ for $5 \mathrm{~min}$ ). The obtained pellet was resuspended and the cell suspension incubated with rat anti-mouse IgG1 microbeads (1:5; Miltenyi Biotec, Germany) in running buffer for 15 min at $4^{\circ} \mathrm{C}$. After two washings with rinsing solution, a maximum of $10^{8}$ cells was resuspended in $500 \mu \mathrm{l}$ of running buffer and processed with the AutoMACs. The p75 LNGFr positive cell fraction was collected.

Schwann cells from adult rat sciatic nerves were isolated according to a technique modified from that of Brockes (70). Animal experimental protocols had been approved previously by the institution's Ethical Committee. Briefly, the sciatic nerves of adult Wistar rat were removed and stored in Hanks' balanced salt solution (Sigma, St. Louis, MO) with $\mathrm{Ca}^{2+}$ and $\mathrm{Mg}^{2+}$ at $0^{\circ} \mathrm{C}$. The epineurium and connective tissue were stripped off with fine forceps, and the nerves were treated with trypsin, collagenase A, and DNase-I for $1 \mathrm{~h}$ with the same steps as explained before. Cells were routinely maintained by plating on poly-L-lysine (100 $\mu \mathrm{g} / \mathrm{ml}$; Sigma, St. Louis, MO)-coated 75 $\mathrm{cm}^{2}$ flasks and this culture medium was replaced every 2 days and cultured at $37^{\circ} \mathrm{C}, 5 \%$ $\mathrm{CO}_{2}$.

Fibroblast cells were obtained from NIH/3T3 fibroblast cell line, purchased from ATCC (Barcelona, Spain, ref CRL-1658).

\subsection{Culture and cell quantification of olfactory ensheathing cells on PCL bars}

A $10 \mu 1$ drop containing $10^{5}$ OECs was deposited on the upper face of the triangular end of the PCL bars. Cells were incubated at $37^{\circ} \mathrm{C}$ and $5 \% \mathrm{CO}_{2}$ for $30 \mathrm{~min}$ to allow adhesion on bars, and afterwards culture medium was added and replaced every 2 days. Studies were performed for different durations $\left(t_{i}\right)$ of $30 \mathrm{~min}, 2,4$ and 7 days. After culture, bars were washed in $0.1 \mathrm{M}$ PBS at $\mathrm{pH} 7.4$ and fixed with 4\% paraformaldehyde in $0.1 \mathrm{M}$ PBS at room temperature for $20 \mathrm{~min}$. Nuclei were counterstained with 4',6-diamidino2-phenylindole dihydrochloride (DAPI, Sigma, 1:5000). OECs were examined using a Leica microscope (DM 6000) and images (10X, from a region with a size of $1.424 \mathrm{~mm}$ width $\times 1.064 \mathrm{~mm}$ length) were obtained with a digital camera (Leica 480X). Eight 
different distances $\left(x_{i}\right)$ along the bars starting from the seeded extreme were evaluated: $x_{i}=1,3,5,10,15,18,20$ and $25 \mathrm{~mm}$, with a number of replicas of $n=4$. For cell quantification, DAPI-labeled cell nuclei were counted at the different chosen distances. Cell number was determined by the free software cellC (71), but corrected taking an average of pixels occupied by a single cell to distinguish clustered cells. In addition, OECs photographs after 30 min of cell culture were taken and cell quantification for this time was considered as the initial condition of the model calculations. Initial cell line density resulted in 75000 cells $/ \mathrm{cm}$ approximately, obtained from the cell number of the drop seeded and the drop's linear extension $(4 \mathrm{~mm})$. For the different distances and times $\left(x_{i}, t_{j}\right)$ the numbers of cells counted on the photographs was averaged to define the experimental cell line density $N^{\text {exp }}\left(x_{i}, t_{j}\right)$.

A similar methodology was employed to determine the maximum distance reached by OECs for each analyzed time. The bar was explored completely using the microscope Leica (DM 6000) and the maximum reached distance was determined as the farthest point on the bar displaying cells. The data of maximum reached distance versus time were fitted to a second order polynomial by a least squares routine. The second order equation was employed to calculate cell speed at different times (2, 4 and 7 days).

\subsection{Immunocytochemistry and scanning electron microscopy}

OECs seeded on the PCL samples were identified by the commonly used p-75, vimentin and S-100 antibodies. Samples were washed with 0.1 M PBS, fixed for $20 \mathrm{~min}$ in 4\% paraformaldehyde, and permeabilized with $0.1 \%(\mathrm{v} / \mathrm{v})$ triton X-100 in $0.1 \mathrm{M}$ PBS for 30 min and to block unspecific binding of the antibodies, samples were incubated with $10 \%(\mathrm{v} / \mathrm{v})$ fetal bovine serum in PBS blocking solution for two hours at room temperature. Afterwards, samples were incubated with monoclonal anti-vimentin (Sigma, 1:40), rabbit anti-S100 antibody (Incstar, Stillwater, MN, ready to use) or mouse anti-low affinity nerve growth factor receptor p-75 (Chemicon, 1:100) and caspase-3 (Abcam, ab32351) overnight at $4^{\circ} \mathrm{C}$ in a humidified chamber; samples were washed again three times in PBS and then incubated with the respective secondary fluorochrome-conjugated antibody (1:200) (goat anti-rabbit Alexa 647 or goat antimouse Alexa 488, Invitrogen) for two hours at room temperature in dark. Finally, samples were washed three times with PBS before mounting the coverslip with a drop 
of vectashield (Vector Laboratories) containing DAPI to counterstain cell nuclei (blue). A Leica TCS SP2 AOBS (Leica Microsystems Heidelberg GmbH, Mannheim, Germany) confocal laser scanning microscope (CLSM) was used.

The morphology of OECs on PCL discs and monofilaments was studied using scanning electron microscopy (SEM). After 4 days in culture, the materials cultured with the OECs were washed in 0.1 M PBS, pH 7.4, and fixed in a 3\% glutaraldehyde solution. Samples were postfixed with $1 \%$ osmium tetroxide, dehydrated in graded ethanol, and coated with gold to be analyzed by SEM (Leica DC300, Bensheim, Germany) at $15 \mathrm{kV}$ and a working distance of $15 \mathrm{~mm}$.

\subsection{Cell viability analysis}

Cell viability was assessed by the MTS assay (CellTiter 96 Aqueous One Solution, Promega) which quantifies mitochondrial activity by measuring the formation of a soluble formazan product which is directly proportional to the number of living cells. After 3, 7 and 10 days of culture for bars and $30 \mathrm{~min}, 2,4$ and 6 days for discs, the materials with the seeded OECs were transferred into a 48-well plate and washed twice with sterile PBS. Culture medium without FBS and phenol red was mixed with MTS in a 5:1 volume ratio, added to the wells, until totally covering the samples, and incubated for $3 \mathrm{~h}$ at $37^{\circ} \mathrm{C}$ in a $5 \% \mathrm{CO}_{2}$ humidified atmosphere in the dark. After the incubation period, $100 \mu \mathrm{l}$ of MTS and medium mixture were transferred into each well of a 96-well plate and absorbance was read in a microplate reader (Victor 3, PerkinElmer) $(n=5)$ at $490 \mathrm{~nm}$.

A MTS assay was performed to compare the cell viability on PCL discs of OECs with those of Schwann cells and fibroblasts. Cells were plated at a density of $2.5 \times 10^{4}$ cells/well in a 48-well culture plate and all three groups (OECs, Schwann cells and fibroblasts) were incubated for $5 \mathrm{~h}, 2,4,6$ and 8 days $(n=3)$. Then the cell medium was removed and replaced by fresh medium without phenol red containing MTS. Cells were then incubated in the dark at $37^{\circ} \mathrm{C}$ for $4 \mathrm{~h} .100 \mu \mathrm{l}$ of each sample solution were transferred to a 96-well plate and absorbance was read in a microplate reader (Victor 3, PerkinElmer) at $490 \mathrm{~nm}$. 


\subsection{OECs net proliferation kinetics}

The cell viability time evolution of the seeded cells was determined and compared in both types of experimental situations, on the discs and bars (from the MTS assay data and the number of cells determined optically by photographing), and modeled as a first order process. The OECs viability on the PCL discs was determined at 4 times $(30 \mathrm{~min}$, 2, 4, and 8 days) and on the PCL bars at three times (3, 7 and 10 days), using the colorimetric MTS assay absorbance data. The $30 \mathrm{~min}$ and 3 days, on discs and on bars respectively, were considered as initial time.

From the number of cells determined optically the viable cells on the PCL bars was calculated as follows: the discrete experimental data $N^{\exp }\left(x_{i}, t_{j}\right)$ for each fixed time $\left(t_{j}=\right.$ 2, 4 and 7 days) were transformed to a continuous function $N\left(x, t_{j}\right)$ giving the cell line density along the bar by interpolating the averaged experimental data at each distance (see below, 2.8.1) with a cubic spline function, using the software Mathcad 2000 (MathSoft, Inc., Cambrige, MA). The interpolated function $N\left(x, t_{j}\right)$ obtained was then integrated between the limits of the region of interest ( 0 to $25 \mathrm{~mm}$ ). Thus, the number of cells $v$ on the bar at each time $t_{j}$ was obtained as

$v\left(t_{j}\right)=\int_{0}^{L} N\left(x, t_{j}\right) d x$

where $L=25 \mathrm{~mm}$. First order kinetics was assumed for OECs net proliferation in both analyses; in each case, the equations employed were

$\ln \left(\frac{A}{A_{0}}\right)=k_{A} \cdot\left(t-t_{0}\right)$ or $\ln \left(\frac{v}{v_{0}}\right)=k \cdot\left(t-t_{0}\right)$

the first equation corresponds to the MTS assay (on discs and on bars) and the second one to the number of cells on the PCL bars calculated by the integration of the experimental data (equation (1)). In equation (2), $A$ is the absorbance at time $t, A_{0}$ is the absorbance at the initial time, $v v$ is the total number of viable cells on the structure at experimental time $t, v_{0}$ is the total number of viable cells on the structure at the initial experimental time $t_{0}$. Data were least-squares fitted for both equations (2) to obtain the net proliferation rates $k_{A}$ and $k$. 


\subsection{Modeling of the colonization patterns}

\subsubsection{Obtaining the experimental line density $N(x, t)$ of cells along the bars}

The colonization by OECs of the bars was modeled as a one-dimensional process, which required to pass from the actual number of cells per unit area obtained experimentally to a consistently defined linear cell density, expressed as number of cells per unit length, $N(x, t)$. Thus, a partial differential equation for $N(x, t)$ along the bar (number of cells per unit bar length at distance $x$, at time $t$ ) governs the process (see below 2.8.2). The experimental data for the linear cell density $N^{\exp }\left(x_{i}, t_{j}\right)$ were obtained from the photographs (see above 2.4) as follows: for each distance $x_{i}$ and time $t_{j}$ the number of cells counted on the four images corresponding to $\left(x_{i}, t_{j}\right)$ was averaged; then, this average was multiplied by the width of the bar $(0.5 \mathrm{~cm})$ and divided by the image area $(0.142 \mathrm{~cm} \times 0.106 \mathrm{~cm})$.

\subsubsection{Diffusion-net proliferation equation}

Cell behavior on the PCL bars was supposed to obey a one-dimensional reactiondiffusion equation with a first order kinetics of cellular net proliferation-and-death term,

$\frac{\partial N}{\partial t}=D \frac{\partial^{2} N}{\partial x^{2}}+k N$

where $N$ is the line density of cells [number of cells $/ \mathrm{cm}$ ], $D$ is the diffusion coefficient $\left[\mathrm{cm}^{2} / \mathrm{min}\right]$ and $k$ is the rate constant for cell net proliferation-and-death [ $\left.\mathrm{min}^{-1}\right]$.

A modification of the above model was also investigated to account for the hypothesis that the populations of cells undergoing proliferation-or-death and migration are disjoint. Consequently, if $N_{m}$ is the line density of migrating cells and $N_{g}$ is the line net density of proliferating cells, one replaces in equation (3) the diffusive term by $D \cdot \partial^{2} N_{m} / \partial x^{2}$ and the source term by $k \cdot N_{g}$. If, further, $\alpha=N_{g} / N$, a population partition coefficient, is assumed to be independent of $(x, t)$, then one has

$\frac{\partial N}{\partial t}=(1-\alpha) D \frac{\partial^{2} N}{\partial x^{2}}+\alpha k N$ 


\subsubsection{Initial and boundary conditions}

Initial and boundary conditions must be supplied to integrate the partial differential equations (3) and (4). Instead of solving a problem corresponding to the actual bar seeded at one end, model calculations were performed for an infinite bar, $-\infty<x<\infty$, seeded at its middle point, $x=0$, and, because of the symmetry of the problem, the results obtained from the model were multiplied by two in order to make them correspond to the real situation for $x \geq 0$. In this way the boundary conditions are simplified. The initial condition was taken as

$$
N(x, 0)= \begin{cases}N_{0} & -r \leq x \leq r \\ 0 & x<-r, x>r\end{cases}
$$

$N_{0}$ was the initial cell line density (obtained from the photograph $30 \mathrm{~min}$ after cell seeding and dividing by the diameter of the seeding drop), and $r$ was the radius of the seeded drop. The boundary conditions at both ends of the infinite bar were set to

$$
\left.\begin{array}{l}
N(-\infty, t)=0 \\
N(\infty, t)=0
\end{array}\right\} \forall t
$$

\subsubsection{Numerical integration}

Equations (3) and (4) with the previously defined initial and boundary conditions, were discretized and solved for fixed values of the model constants, $(D, k)$ or $(D, k, \alpha)$, respectively, by numerical methods using the software Mathcad 2000. The model characteristics, satisfying the stability condition, were: 200 partitions of the length, each $0.1 \mathrm{~cm}$ long, and 480 time partitions, with intervals of $30 \mathrm{~min}$.

In the model implementation cells were assumed to be seeded in the centre of a $20 \mathrm{~cm}$ long one-dimensional line, a much longer line than the actual geometry $(3 \mathrm{~cm})$ in order to guarantee that the imposed boundary conditions did not influence the solutions obtained in the region of interest (the actual $3 \mathrm{~cm}$ length).

The first implemented model (equation 3) was solved for $D$ and $k$ values and these were optimized by minimizing the mean quadratic error between experimental and model results, 
$E R R O R=\sqrt{\frac{\sum_{i, j}\left(N\left(x_{i}, t_{j}\right)-N^{\exp }\left(x_{i}, t_{j}\right)\right)^{2}}{i \cdot j}}$

where $N\left(x_{i}, t_{j}\right)$ is the cell line density determined from the model [number of cells $/ \mathrm{cm}$ ] for a pair $(D, k), N^{e x p}\left(x_{i}, t_{j}\right)$ is the cell line density from experimental data; $i$ is the index labeling points along the model line, and $j$ is the index labeling instants of time.

Equation (4) was discretized and implemented by numerical methods in a similar way. In order to study the separate effect of each phenomenon, each parameter was independently varied keeping the rest fixed, and plotting the obtained solution. The more convenient parameters of model 2 were chosen from this study.

\subsection{Cell aspect ratio and cell alignment analysis}

In order to characterize the elongated shape acquired by cells cultured on the PCL filaments the aspect ratio (length divided by width) of the cells and the angle formed by the axis of their longest dimension with the PCL filament axis were measured as follows. On two confocal images (40 times magnification) of PCL filaments with cells cultured for 4 days ten cells were selected on each filament, and with the help of the ImageJ software (Java-based image processing program developed at the National Institutes of Health, USA) were measured the length and width of the cell shapes; "length" was taken as the value of the cell's largest linear dimension, "width" was then taken as the value of the largest linear measure perpendicular to "length". For the same cell shapes the angle between the "length" dimension and the filament axis was also determined with the same software.

\subsection{Statistics}

All the experimental data were presented as means \pm standard deviation. Statistical assessment of significant variance was performed using a one-way ANOVA with a Tukey multiple range test by the software Statgraphics plus (Statistical graphics corp. v. 5.1 Princeton, NJ). Statistical tests were performed at a 95\% significance level ( $p$ value $<0.05)$. 


\section{Results}

\subsection{OECs viability and morphology on PCL substrata}

The olfactory ensheathing cells were viable on PCL materials and their number increased steadily with culture time, as revealed by the MTS assay. OECs proliferate on PCL at a rate qualitatively similar to that of Schwann cells, and, expectedly, significantly slower than fibroblasts (which were taken as comparison control cells), Figure 2.

The results of immunocytochemistry showed an excellent colonization by OECs of the PCL flat surface (Figure 3). After 4 days, primary OECs expressed the markers S100 and vimentin (Figure 3A and 3D). The OECs covered the totality of substrate surface, indicating that PCL favors cell adhesion and proliferation. The proportion of positive cells was high, showing that the purified cells maintained their phenotype in culture on PCL. The presence of active caspase-3 positive cells undergoing apoptosis process was rarely observed (Figure 3C). These results support other evidence $(72,73)$ on PCL as a suitable substrate promoting cell attachment and proliferation for glial cells.

Figure 4A shows the electron micrograph of a (non-seeded) PCL monofilament and Figures $4 \mathrm{C}$ and D show the OECs on the 80 micron diameter PCL monofilaments after 4 days of culture. The drawing stage in the process of manufacture of the PCL monofilaments produced a pattern of microgrooves on the surface of the fiber, clearly observable in Figure 4A. PCL is a semicrystalline polymer, in which amorphous regions constituted by disordered chains alternate with the regularly packed chains of the polymer crystallites. Upon stretching, microcrystallite orientation, interstitial chain alignment and cold crystallization take place, giving rise to an observable microgrooved surface pattern.

OECs colonized and completely covered the length of the filaments, with the cell cytoplasm adapting to the surface's curvature, and with the cytoplasm of adjacent cells touching each other through processes. OECs formed an interconnected network of almost parallel linear cells arrays with a preferential alignment along the filament axis 
and some circumferential spacing, the cells adopting an elongated fusiform and bipolar morphology. The patterned surface microtopography of the monofilaments may be influential on this shape: the fiber surface is constituted by oriented alternating amorphous and crystalline domains, each with different rigidities, giving rise to a preferentially oriented microgrooved topography to which the cell transmembrane's proteins involved in migration may be sensitive. Measurements of the aspect ratio (length/width) of the cells gave a figure of $9.8 \pm 4.3 \mu \mathrm{m}$; that is, in the mean, cells were

almost 10 times longer than wide on the filament. The mean length and width of the cells were $73.8 \pm 18.2 \mu \mathrm{m}$ and $7.5 \pm 2.4 \mu \mathrm{m}$, respectively. They were also polarized along it: measurements of the angle between the cells' axis and the filament's axis gave a value of $4.2 \pm 3.5$ degrees, showing their high degree of alignment (Figure 4B).

The morphology of OECs on the different substrata was also assessed with scanning electron microscopy. The micrographs (Figure 5A, B) show that OECs adhered to the substrata exhibited different morphologies on PCL filaments and discs: spindle-shaped cells were observed extending processes along the filaments (Figure 5A), with a bipolar morphology typical of a cell in migration, whereas more flattened multipolar cells were spread on the discs (Figure 5B).

\subsection{OECs colonization of PCL bars}

OECs, once seeded initially on one of the bar's ends, were subsequently able to migrate along and colonize the whole length of the bar in 7 days (Figure 6). After 2 days, at 1 $\mathrm{mm}$ distance from the seeded end the cell density had decreased considerably with respect to the initial time as a consequence of the cell migration. Generally, at subsequent times the cell density grew for each distance and the maximal reached distance increased.

The advance front of OECs on the bars (maximal distance reached by any cell) for each time was determined by optical inspection of the photographs, and is given on Figure 7. The advance front thus defined does not have a constant speed, but seems to be slower with time (a second order polynomial describes satisfactorily the maximum distance versus time curve). 


\subsection{One-dimensional modeling of the colonization pattern of OECs on PCL bars}

For each pair $(D, k)$ equation (3) can be numerically solved and the colonization patterns $N(x, t), 0 \leq x \leq L$, can be compared, for each $t$, with the experimentally found data, once these are converted to a line density profile of cells. Least-squares minimization of the discrepancy gives a pair of parameter values, which in our case were $D=1.95 \cdot 10^{-5} \mathrm{~cm}^{2} / \min \left[3.25 \cdot 10^{-5} \mathrm{~mm}^{2} / \mathrm{s}\right]$ and $k=7.97 \cdot 10^{-5} \mathrm{~min}^{-1}\left[1.33 \cdot 10^{-6}\right.$ $\mathrm{mm}^{2} / \mathrm{s}$ ]. The behavior predicted by the model equations with these parameter values yielded the approximation to the experimental data shown in Figure 8.

The model was able to reproduce qualitatively two patterns exhibited by the experimental data: the time oscillations of the cell density at fixed distances (see Figure 6), and the increasing spread of cells along the $\mathrm{x}$-axis as time increased (see Figure 7). Quantitatively, the model predictions with the least-squares optimized parameters were better at the shorter distances, and were much poorer for the longer distances, the more so the longer the time.

\subsection{OECs net proliferation kinetics}

As explained above, a first order relationship was assumed to describe the net proliferation kinetics of the OECs on the PCL bars. The consistency of this assumption with the independent MTS data obtained on PCL discs and bars can be ascertained on Figure 9, where the three sets of data, the MTS absorbance data on bars, on discs, and the integrated experimental total cell number data, $v$, from the raw information stemming from photograph counting (see section 2.7, equation (1)), have been represented in a logarithm versus time plot.

The linearity of each of the dependences is satisfactory enough. Linear fits gave for the net proliferation rate $k$ values of $8.04 \cdot 10^{-5} \mathrm{~min}^{-1}$ on the PCL discs (MTS assay, Rsquared 0.921 ), $1.93 \cdot 10^{-4} \mathrm{~min}^{-1}$ on the PCL bars (MTS assay, R-squared 0.858), and $1.27 \cdot 10^{-4} \mathrm{~min}^{-1}$ (R-squared 0.951 ) from the integration of the experimental data. Even though they stem from such different experiments, the three are of comparable order of magnitude. 


\subsection{Assessment of the separate qualitative influence on colonization patterns of diffusivity and net proliferation-and-death}

Figure 10 shows the results of solving equation (4) changing the value of one parameter while the others were kept fixed with the values obtained from the fitting of equation (3). The diffusion coefficient $D$ was studied for 4 different values, $4 \cdot 10^{-7}, 4 \cdot 10^{-6}, 4 \cdot 10^{-5}$ and $4 \cdot 10^{-4} \mathrm{~cm}^{2} / \mathrm{min}$, while keeping the net proliferation rate and the partition coefficient fixed at $k=7.97 \cdot 10^{-5} \mathrm{~min}^{-1}$ and $\alpha=0.6$, respectively. Larger values of $D$ result in a faster colonization of the bar, and in a correspondingly lower number of cells at shorter distances from the seeding point as time progresses. Lower diffusivities lead to a steeper gradient of cell concentration along the bar, and to a cumulative increase of cells near the seeded end as time progresses. Thus, a crossover effect occurs at short distances as a function of cell diffusivity.

Any such nonlinearity is absent from the influence of the net proliferation rate $k$. Values of $k=1 \cdot 10^{-5}, 5 \cdot 10^{-5}, 1 \cdot 10^{-4}$ and $2 \cdot 10^{-4} \mathrm{~min}^{-1}$ were studied keeping $D$ and $\alpha$ fixed at $1.95 \cdot 10^{-5} \mathrm{~cm}^{2} / \mathrm{min}$ and 0.6 , respectively. An increase in the proliferation rate has always the effect of augmenting the number of cells proportionally to cell density for all distances and times, with differences increasing throughout time.

Finally, the partition coefficient $\alpha$ was varied as $0.2,0.4,0.6$ and 0.8 for constant values of the diffusion coefficient and net proliferation rate of $1.95 \cdot 10^{-5} \mathrm{~cm}^{2} / \mathrm{min}$ and $7.97 \cdot 10^{-5}$ $\min ^{-1}$, respectively. As expected, the value of $\alpha$ combines the qualitative patterns of colonization ascribable to $D$ and $k$ separately: the lower $\alpha$, the more the qualitative trends of colonization are governed by migrational features; the larger $\alpha$, the more they are determined by proliferative behavior.

\section{Discussion}

The ultimate goal of a targeted induced growth of axons across long distances puts a need on the development of synthetic structures to help this process. Thread-like materials covered with specialized cells such as OECs may represent such a guidance bed for neuronal axons. We have shown here that OECs can colonize the PCL surfaces 
keeping their distinctive phenotypic features, both on flat discs and on structures having long aspect ratio (microfilaments and bars). On PCL microgrooved filaments OECs adopt a clearly bipolar shape and interact forming chains, whereas on flat substrata with the same composition they exhibit a variety of shapes, from bipolar (now without any directional preference) to tripolar and rounded with lamellipodia.

In the mammalian olfactory nervous system OECs show a variety of morphologies,, which have been related to their different functionalities $(74,75)$. OEC is a malleable type of cell which can exhibit different cytoskeletal organizations: on one hand, OECs possessing a long fusiform bipolar morphology, which have been called Schwann-like OECs, and on the other hand cells with flat sheet-like morphology with more or less centered nuclei, called astrocyte-like OECs. These subpopulations transform into each other in response to a variety of environmental stimuli (such as extracellular factors or interactions with a variety of cells) with mechanisms to date largely unknown. This difference in cytoskeleton organization correlates with different migratory properties from the nasal epithelium to the olfactory nerve, and different functionalities and expression of growth-promoting molecules. Schwann-like olfactory ensheathing cells contact each other and extend processes ahead of the growing axons, whose bundles they compartmentalise and ensheath from their emergence in the nasal epithelium. In the axonal path towards the olfactory bulb, cells acquire a more flattened shape, astrocyte-like, more loosely associated to the others, leading to defasciculation and sorting of axons in those expressing the same odorant receptors $(74,75)$.

We hypothesize that the parallely microgrooved patterned structure of the filaments' surface induces the fusiform cytoskeletal organization here observed on filaments, which contrasts with the cell morphology on the flat, isotropous PCL disk surfaces. The cell transmembrane proteins responsible for the cytoskeleton development and organization are sensitive to the regularly alternating pattern at the nanometer scale. A similar effect was reported in (35), where the authors found a dependence of cell shape and alignment on the dimensions of electrospun silk fibroin fibres.

OECs are known to express molecules such as BDNF, GDNF or NGF that regulate their migration and stimulate axonal regeneration $(75,76)$. This fact, along with the ability of polymeric microfilaments to induce the OEC migratory phenotype, makes one hope that 
this material-cell combination serve as a suitable substrate to enhance axonal growth along them.

The colonization pattern (the number of cells at each position and for each time subsequent to cell seeding at one extreme) can be characterized by a cell line density function $N(x, t)$ and its changes with $x$ and with $t$. To the number of cells seen at aiven time and a at given position contribute both the rate at which cells fixed at that position are proliferating and dying, and the cells which are migrating to that position from other neighbouring points, and thus the colonization patterns are due to both the migration and the proliferation-and-death of the cells. The images, however, cannot discriminate the contributions from both phenomena to the overall pattern, and in order to have a qualitative picture of the complex phenomenon the modelling of the process was undertaken.

The colonization of the bars was assimilated to a spatially one-dimensional process, with the bar modelled as a line, $0 \leq x \leq L$, and with a cell line density $N(x, t)$ (number of cells per unit length) varying at each point $x$ along the line with time as a consequence of migration and of proliferation-and-death; only a net source term (proliferation minus death) was considered. If the first is assumed to be a Fickian process (unbiased drift in opposite directions, independent of position) and the latter is assumed to be proportional to $N$, then a mass balance delivers equation (3). The diffusivity $D$ and rate parameter $k$ obtained by a least-squares fit of the model describe qualitatively the experimental behaviour (Figure 8), though discrepancies between the model prediction and the experimental colonization patterns are noticeable. Possible sources for the model's mismatches can be roughly classified into two groups. On the one hand, there is the uncertainty of the experimental results, which is addressed below. On the other hand, one may question and modify the basic hypotheses on which equation (3) relies. The constancy assumed for both the diffusivity $D$ and the proliferation-and-death rate $k$ represents an important simplification, which delivers a linear model equation and thus simplifies its numerical solution; this is why these assumptions are almost universally adopted in the literature. A more realistic consideration of the problem would probably need to contemplate a dependence of $D$ and/or of $k$ on factors of their local environment: for example, clustering of cells might alter nutrient access or metabolite diffusion or biochemical interactions apart from those with the material that may affect their motility, and thus alter their migrating/proliferating relative probability. This 
would entail a dependence of the diffusivity and the net rate of proliferation-and-death on the position coordinate, $D=D(x), k=k(x)$. This alternative wasn't considered here because it would have complicated the model unnecessarily. Another hypothesis of equation (3) is the assumed first-order net proliferation-and-death kinetics. Higher order kinetics, or more involved relationships, could have been employed instead; however, the experimental findings could in our case be consistently accounted for by a firstorder kinetics (see section 3.5).

Still another hypothesis of equation (3) which can be modified is the independence it presupposes for the two cell-fate events of migration and proliferation; this is certainly not true, since cells which are migrating do not proliferate-or-die. In order to take this fact into account, in equation (4) the total cell density $N$ was split into those cells which proliferate-or-die, $\alpha \cdot N$, and those which migrate, $(1-\alpha) \cdot N$; for simplicity, the partition coefficient of both populations, $\alpha$, was assumed to be independent of $(x, t)$. Equation (4) is a finer treatment of the problem than is equation (3) in that it involves the correlation of these cell events; however, from the analytical point of view both equations are the same, equation (4) being equivalent to equation (3) with different effective parameters, $D^{\prime}=(1-\alpha) \cdot D$ and $k^{\prime}=\alpha \cdot k$. Because of this, instead of least-squares optimizing the set of the three free parameters $(D, k, \alpha)$ of equation (4) to compare the predicted curves with the experimental data, it was decided to study their independent influence on the colonization patterns by keeping two of them fixed and changing the third, and doing this for each one of the three parameters; values of $D$ and $k$ were changed around their optimized values in equation (3). Figure 10 shows the results of the trials. This qualitative analysis of the parameters influence can suggest forms of distance dependences for $D, k$ and $\alpha$ which would improve the model's reproduction of the experimental behavior; in the absence of more concrete theoretical reasons for those $\mathrm{x}$ dependences, however, their incorporation into the model would be gratuitous and an unnecessary complication. Still, the above parameter analysis guides a choice of constant values for $(D, k, \alpha)$ capable to smooth out some of the more salient discrepancies between the experimental data and the model's prediction. Thus, if high values for the net proliferation-and-death rate $\left(k=1.7 \cdot 10^{-4} \mathrm{~min}^{-1}\right)$, partition coefficient $(\alpha=0.8)$ and diffusivity $\left(D=2.53 \cdot 10^{-4} \mathrm{~cm}^{2} / \mathrm{min}\right.$ ) are adopted, the pattern predicted by equation (4) shows an improvement over equation (3), in that now the mismatches at the shorter and the longer distances have been greatly reduced (see Figure 8). 
Experimentally determined diffusion coefficients for various cell types in a range of $1.6 \cdot 10^{-6}$ to $1.2 \cdot 10^{-5} \mathrm{~mm}^{2} / \mathrm{s}$ have been reported in the literature (53). In a purely diffusive Brownian process the mean squared distance $\left\langle x^{2}\right\rangle$ travelled by a particle after a time $t$ is related to the diffusion coefficient $D$ by $\left\langle x^{2}\right\rangle=2 \cdot d \cdot D \cdot t$, with $\mathrm{d}$ the dimensionality of space (77). Putting here $d=1, D=2.53 \cdot 10^{-4} \mathrm{~cm}^{2} / \mathrm{min}$ and $t=7$ days one obtains $\sqrt{ }\left\langle x^{2}\right\rangle=$ $22.6 \mathrm{~mm}$, startlingly close to the experimentally found approximate value of $24.5 \mathrm{~mm}$ reached by the cells on the bars at that time (Figure 7). In Fisher's model $(66,78)$ the front of advance has a constant speed of $2 \cdot \sqrt{ }(D \cdot k)$; for the above values of $D$ and $k$ this expression gives $6.913 \cdot 10^{-5} \mathrm{~mm} / \mathrm{s}$, whereas our experimental mean speed of advance (from the data of Fig. 7) varies between $6.15 \cdot 10^{-5}$ and $3.09 \cdot 10^{-5} \mathrm{~mm} / \mathrm{s}$. From Figure $5 \mathrm{E}$ of reference (35), a value of $1.25 \cdot 10^{-5} \mathrm{~mm} / \mathrm{s}$ can be estimated for OECs migration speed on silk fibroin fibres.

The values of $D$ and $k$ are the summaries in this highly simplified model of the phenomena of proliferation-and-death and migration, which determine the colonization pattern of our system. A change in the chemistry of the material substrate or in the type of material guidance might easily alter the weight of both factors, and thus lead to different colonization patterns. It is in considering such possible different combinations that the modeling parameters may be of help for characterizing, and then predicting, the behaviour of OECs on alternative types of constructs.

Notwithstanding the semiquantitative insights provided by this analysis, some discrepancies between the prediction and the experimental data still persist, maybe due to the unavoidable variability of biological behaviour of each sample, and to the nature of the one-dimensional approximation of the phenomenon, which necessitates the definition of averaged cell line densities from the photographs, as described in 2.8.1, with the unavoidable counting uncertainties, and, moreover, it disregards the fraction of cells which has migrated at places out of reach of the microscope's camera.

\section{Conclusions}

Olfactory ensheathing cells are able to migrate on and colonize long filaments and bars of polycaprolactone maintaining the phenotype. The cells migrate over the cylindrical surface of the filaments giving rise to highly aligned arrays of cells. In order to 
quantitatively assess this process, an experimental set-up forcing cells to spread along narrow long PCL bars was implemented, and the most salient features of the pattern of invasion could be characterized with the three parameters of a simple reaction-diffusion differential model: a coefficient of diffusion (or migration), a rate of net proliferationand-death, and a partition of the cell population into a fraction migrating and a fraction not migrating. The values obtained from parameter fitting are in the correct orders of magnitude, and allow for an analytical insight into the colonization process which distinguishes between proliferation-or-death events and migration events. The speed of the invasion front could also be experimentally measured and compared with the one predicted by the model. For this type of cell, in this one-dimensional guiding environment and on this kind of material substrate, one must conclude that the net proliferative kinetics slightly overweighs the migrational propensity in determining the pattern of invasion.

Acknowledgments. Support of the Spanish Science \& Innovation Ministery through project MAT2008-06434 is acknowledged. MMP and CMR acknowledge partial funding through the "Convenio de Colaboración para la Investigación Básica y Traslacional en Medicina Regenerativa" between the Instituto Nacional de Salud Carlos III, the Conselleria de Sanidad of the Generalitat Valenciana, and the Foundation Centro de Investigación Principe Felipe. JLEI acknowledges the support of the Spanish Science \& Innovation Ministry through the "Campus de Excelencia Internacional" program with the Universitat Politècnica de València. 


\section{References}

1. Stokols S, Sakamoto J, Breckon C, Holt T, Weiss J, Tuszynski MH. Templated agarose scaffolds support linear axonal regeneration. Tissue Eng. 2006;12(10):2777-87. 2. Wei YT, Tian WM, Yu X, Cui FZ, Hou SP, Xu QY, et al. Hyaluronic acid hydrogels with IKVAV peptides for tissue repair and axonal regeneration in an injured rat brain. Biomed Mater. 2007;2(3):142-6.

3. Yao L, Wang S, Cui W, Sherlock R, O'Connell C, Damodaran G, et al. Effect of functionalized micropatterned PLGA on guided neurite growth. Acta Biomater. 2009;5(2):580-8.

4. Chehrehasa F, Windus LCE, Ekberg JAK, Scott SE, Amaya D, Mackay-Sim A, et al. Olfactory glia enhance neonatal axon regeneration. Molecular and Cellular Neuroscience. 2010;45(3):277-88.

5. Chen BK, Knight AM, de Ruiter GC, Spinner RJ, Yaszemski MJ, Currier BL, et al. Axon regeneration through scaffold into distal spinal cord after transection. J Neurotrauma. 2009;26(10):1759-71.

6. Goto E, Mukozawa M, Mori H, Hara M. A rolled sheet of collagen gel with cultured Schwann cells: model of nerve conduit to enhance neurite growth. J Biosci Bioeng. 2010;109(5):512-8.

7. Lietz M, Dreesmann L, Hoss M, Oberhoffner S, Schlosshauer B. Neuro tissue engineering of glial nerve guides and the impact of different cell types. Biomaterials. 2006;27(8):1425-36.

8. Radtke C, Sasaki M, Lankford KL, Vogt PM, Kocsis JD. Potential of olfactory ensheathing cells for cell-based therapy in spinal cord injury. J Rehabil Res Dev. 2008;45(1):141-51.

9. Wei Y, Miao X, Xian M, Zhang C, Liu X, Zhao H, et al. Effects of transplanting olfactory ensheathing cells on recovery of olfactory epithelium after olfactory nerve transection in rats. Med Sci Monit. 2008;14(10):198-204.

10. Tennent R, Chuah MI. Ultrastructural study of ensheathing cells in early development of olfactory axons. Brain Res Dev Brain Res. 1996;95(1):135-9.

11. Doucette R. Glial influences on axonal growth in the primary olfactory system. Glia. 1990;3(6):433-49.

12. Field P, Li Y, Raisman G. Ensheathment of the olfactory nerves in the adult rat. J Neurocytol. 2003 Mar;32(3):317-24. 
13. Boyd JG, Doucette R, Kawaja MD. Defining the role of olfactory ensheathing cells in facilitating axon remyelination following damage to the spinal cord. Faseb J. 2005;19(7):694-703.

14. Franklin RJ, Gilson JM, Franceschini IA, Barnett SC. Schwann cell-like myelination following transplantation of an olfactory bulb-ensheathing cell line into areas of demyelination in the adult CNS. Glia. 1996;17(3):217-24.

15. Imaizumi T, Lankford KL, Waxman SG, Greer CA, Kocsis JD. Transplanted olfactory ensheathing cells remyelinate and enhance axonal conduction in the demyelinated dorsal columns of the rat spinal cord. J Neurosci. 1998;18(16):6176-85.

16. Raisman G. Olfactory ensheathing cells - another miracle cure for spinal cord injury? Nat Rev Neurosci. 2001;2(5):369-75.

17. Ramón-Cueto A, Cordero MI, Santos-Benito FF, Avila J. Functional recovery of paraplegic rats and motor axon regeneration in their spinal cords by olfactory ensheathing glia. Neuron. 2000;25(2):425-35.

18. Chuah MI, Choi-Lundberg D, Weston S, Vincent AJ, Chung RS, Vickers JC, et al. Olfactory ensheathing cells promote collateral axonal branching in the injured adult rat spinal cord. Exp Neurol. 2004;185(1):15-25.

19. Bellamkonda RV. Peripheral nerve regeneration: an opinion on channels, scaffolds and anisotropy. Biomaterials. 2006;27(19):3515-8.

20. Liu Y, Gong Z, Liu L, Sun H. Combined effect of olfactory ensheathing cell transplantation and glial cell line-derived neurotrophic factor (GDNF) intravitreal injection on optic nerve injury in rats. Molecular Vision. 2010;16:2903-10.

21. Zhu Y, Cao L, Su Z, Mu L, Yuan Y, Gao L, et al. Olfactory ensheathing cells: attractant of neural progenitor migration to olfactory bulb. Glia. 2010;58(6):716-29.

22. Basiri M, Doucette R. Sensorimotor cortex aspiration: a model for studying Wallerian degeneration-induced glial reactivity along the entire length of a single CNS axonal pathway. Brain Res Bull. 2010;81(1):43-52.

23. Li Y, Carlstedt $\mathrm{T}$, Berthold $\mathrm{C}-\mathrm{H}$, Raisman $\mathrm{G}$. Interaction of transplanted olfactory-ensheathing cells and host astrocytic processes provides a bridge for axons to regenerate across the dorsal root entry zone. Experimental Neurology. 2004;188(2):3008.

24. Li Y, Yamamoto M, Raisman G, Choi D, Carlstedt T. An experimental model of ventral root repair showing the beneficial effect of transplanting Olfactory Ensheathing Cells. Neurosurgery. 2007;60(4):734-41. 
25. Ramón-Cueto A, Plant GW, Avila J, Bunge MB. Long-distance axonal regeneration in the transected adult rat spinal cord is promoted by olfactory ensheathing glia transplants. J Neurosci. 1998;18(10):3803-15.

26. Gómez-Pinedo U, Vidueira S, Sancho FJ, García-Verdugo JM, Matías-Guiu J,Barcia JA. Olfactory ensheathing glia enhances reentry of axons into the brain from peripheral nerve grafts bridging the substantia nigra with the striatum. Neurosci Lett. 2011;494(2):104-8.

27. Graziadei PP, Levine RR, Graziadei GA. Regeneration of olfactory axons and synapse formation in the forebrain after bulbectomy in neonatal mice. Proc Natl Acad Sci USA. 1978;75(10):5230-4.

28. Cao L, Liu L, Chen ZY, Wang LM, Ye JL, Qiu HY, et al. Olfactory ensheathing cells genetically modified to secrete GDNF to promote spinal cord repair. Brain. 2004;127(3):535-49.

29. Cao L, Su Z, Zhou Q, Lv B, Liu X, Jiao L, et al. Glial cell line-derived neurotrophic factor promotes olfactory ensheathing cells migration. Glia. 2006;54(6):536-44.

30. Woodhall E, West AK, Chuah MI. Cultured olfactory ensheathing cells express nerve growth factor, brain-derived neurotrophic factor, glia cell line-derived neurotrophic factor and their receptors. Brain Res Mol Brain Res. 2001;88(1-2):203-13.

31. Cao L, Zhu YL, Su Z, Lv B, Huang Z, Mu L, et al. Olfactory ensheathing cells promote migration of Schwann cells by secreted nerve growth factor. Glia. 2007;55(9):897-904.

32. Doucette R. Immunohistochemical localization of laminin, fibronectin and collagen type IV in the nerve fiber layer of the olfactory bulb. Int $\mathrm{J}$ Dev Neurosci. 1996;14(7-8):945-59.

33. Franceschini IA, Barnett SC. Low-affinity NGF-receptor and E-N-CAM expression define two types of olfactory nerve ensheathing cells that share a common lineage. Dev Biol. 1996;173(1):327-43.

34. Runyan SA, Phelps PE. Mouse olfactory ensheathing glia enhance axon outgrowth on a myelin substrate in vitro. Exp Neurol. 2009;216(1):95-104.

35. Shen Y, Qian Y, Zhang H, Zuo B, Lu Z, Fan Z, et al. Guidance of olfactory ensheathing cell growth and migration on electrospun silk fibroin scaffolds. Cell Transplant. 2010;19(2):147-57. 
36. Li B-C, Jiao S-S, Xu C, You H, Chen J-M. PLGA conduit seeded with olfactory ensheathing cells for bridging sciatic nerve defect of rats. J Biomed Mater Res-A. 2010;94(3):769-80.

37. Clements IP, Kim Y-t, English AW, Lu X, Chung A, Bellamkonda RV. Thinfilm enhanced nerve guidance channels for peripheral nerve repair. Biomaterials. 2009;30(23-24):3834-46.

38. Martín-López E, Nieto-Díaz M, Nieto-Sampedro M. Differential adhesiveness and neurite-promoting activity for neural cells of chitosan, gelatin, and poly-L-lysine films. J Biomater Appl. 2012;26(7):791-809.

39. Cai J, Peng X, Nelson KD, Eberhart R, Smith GM. Permeable guidance channels containing microfilament scaffolds enhance axon growth and maturation. J Biomed Mater Res A. 2005;75(2):374-86.

40. Novikova LN, Mosahebi A, Wiberg M, Terenghi G, Kellerth JO, Novikov LN. Alginate hydrogel and matrigel as potential cell carriers for neurotransplantation. $\mathbf{J}$ Biomed Mater Res-A. 2006;77(2):242-52.

41. Tang ZP, Liu N, Li ZW, Xie XW, Chen Y, Shi YH, et al. In vitro evaluation of the compatibility of a novel collagen-heparan sulfate biological scaffold with olfactory ensheathing cells. Chin Med J (Engl). 2010;123(10):1299-304.

42. Wang B, Zhao Y, Lin H, Chen B, Zhang J, Zhang J, et al. Phenotypical analysis of adult rat olfactory ensheathing cells on 3-D collagen scaffolds. Neurosci Lett. 2006;401(1-2):65-70.

43. Guarnieri D, De Capua A, Ventre M, Borzacchiello A, Pedone C, Marasco D, et al. Covalently immobilized RGD gradient on PEG hydrogel scaffold influences cell migration parameters. Acta Biomater. 2010;6(7):2532-9.

44. Ngo TT, Waggoner PJ, Romero AA, Nelson KD, Eberhart RC, Smith GM. Poly(L-Lactide) microfilaments enhance peripheral nerve regeneration across extended nerve lesions. J Neurosci Res. 2003;72(2):227-38.

45. Schnell E, Klinkhammer K, Balzer S, Brook G, Klee D, Dalton P, et al. Guidance of glial cell migration and axonal growth on electrospun nanofibers of poly-ecaprolactone and a collagen/poly-e-caprolactone blend. Biomaterials. 2007;28(19):3012-25.

46. Lim SH, Liu XY, Song H, Yarema KJ, Mao HQ. The effect of nanofiber-guided cell alignment on the preferential differentiation of neural stem cells. Biomaterials. 2010;31(34):9031-9. 
47. Wong DY, Hollister SJ, Krebsbach PH, Nosrat C. Poly(epsilon-caprolactone) and poly (L-lactic-co-glycolic acid) degradable polymer sponges attenuate astrocyte response and lesion growth in acute traumatic brain injury. Tissue Eng. 2007;13(10):2515-23.

48. Wong DY, Krebsbach PH, Hollister SJ. Brain cortex regeneration affected by scaffold architectures. J Neurosurg. 2008;109(4):715-22.

49. Wong DY, Leveque JC, Brumblay H, Krebsbach PH, Hollister SJ, Lamarca F. Macro-architectures in spinal cord scaffold implants influence regeneration. $\mathrm{J}$ Neurotrauma. 2008;25(8):1027-37.

50. Pierucci A, de Duek EA, de Oliveira AL. Peripheral nerve regeneration through biodegradable conduits prepared using solvent evaporation. Tissue Eng Part A. 2008;14(5):595-606.

51. Vleggeert-Lankamp CL, de Ruiter GC, Wolfs JF, Pego AP, van den Berg RJ, Feirabend HK, et al. Pores in synthetic nerve conduits are beneficial to regeneration. J Biomed Mater Res-A. 2007;80(4):965-82.

52. Cai AQ, Landman KA, Hughes BD. Multi-scale modeling of a wound-healing cell migration assay. J Theor Biol. 2007;245(3):576-94.

53. Maini PK, McElwain DL, Leavesley DI. Traveling wave model to interpret a wound-healing cell migration assay for human peritoneal mesothelial cells. Tissue Eng. 2004;10(3-4):475-82.

54. Dokukina IV, Gracheva ME. A Model of Fibroblast Motility on Substrates with Different Rigidities. Biophys J. 2010;98(12):2794-803.

55. Schneider IC, Haugh JM. Spatial Analysis of 3' Phosphoinositide Signaling in Living Fibroblasts: II. Parameter Estimates for Individual Cells from Experiments. Biophys J. 2004;86(1):599-608.

56. Marcy Y, Prost J, Carlier M-F, Sykes Cc. Forces generated during actin-based propulsion: A direct measurement by micromanipulation. PNAS. 2004;101(16):5992-7.

57. Mogilner A, Oster G. Polymer motors: pushing out the front and pulling up the back. Curr Biol. 2003;13(18):R721-33.

58. Cheng G, Youssef BB, Markenscoff P, Zygourakis K. Cell population dynamics modulate the rates of tissue growth processes. Biophys J. 2006;90(3):713-24.

59. Galbusera F, Cioffi M, Raimondi MT, Pietrabissa R. Computational modeling of combined cell population dynamics and oxygen transport in engineered tissue subject to interstitial perfusion. Comput Methods Biomech Biomed Engin. 2007;10(4):279-87. 
60. Hatzikirou H, Deutsch A. Cellular automata as microscopic models of cell migration in heterogeneous environments. Curr Top Dev Biol. 2008;81:401-34.

61. Reffay M, Petitjean L, Coscoy S, Grasland-Mongrain E, Amblard F, Buguin A, et al. Orientation and polarity in collectively migrating cell structures: statics and dynamics. Biophys J. 2011;100(11):2566-75.

62. Chung CA, Yang CW, Chen CW. Analysis of cell growth and diffusion in a scaffold for cartilage tissue engineering. Biotechnol Bioeng. 2006;94(6):1138-46.

63. Dunn JC, Chan WY, Cristini V, Kim JS, Lowengrub J, Singh S, et al. Analysis of cell growth in three-dimensional scaffolds. Tissue Eng. 2006;12(4):705-16.

64. Harms BD, Bassi GM, Horwitz AR, Lauffenburger DA. Directional persistence of EGF-induced cell migration is associated with stabilization of lamellipodial protrusions. Biophys J. 2005;88(2):1479-88.

65. Lemon G, King J. Travelling-wave behaviour in a multiphase model of a population of cells in an artificial scaffold. J Math Biol. 2007;55(4):449-80.

66. Fisher R. The wave of advance of advantageous genes. Ann Eugenics. 1937;7:355-69.

67. Graner Fo, Glazier JA. Simulation of biological cell sorting using a twodimensional extended Potts model. Phys Rev Lett. 1992;69(13):2013-6.

68. Ouaknin GY, Bar-Yoseph PZ. Stochastic Collective Movement of cells and fingering morphology: no maverick cells. Biophys J. 2009;97(7):1811-21.

69. Savill NJ, Hogeweg P. Modelling morphogenesis: from single cells to crawling slugs. J Theor Biol. 1997;184(3):229-35.

70. Brockes JP, Fields KL, Raff MC. Studies on cultured rat Schwann cells. I. Establishment of purified populations from cultures of peripheral nerve. Brain Res 1979;165:105-118.

71. Selinummi J, Seppala J, Yli-Harja O, Puhakka JA. Software for quantification of labeled bacteria from digital microscope images by automated image analysis. Biotechniques. 2005;39(6):859-63.

72. Gupta D, Venugopal J, Prabhakaran MP, Dev VR, Low S, Choon AT, et al. Aligned and random nanofibrous substrate for the in vitro culture of Schwann cells for neural tissue engineering. Acta Biomater. 2009;5(7):2560-9.

73. Nisbet DR, Yu LM, Zahir T, Forsythe JS, Shoichet MS. Characterization of neural stem cells on electrospun poly(epsilon-caprolactone) submicron scaffolds: 
evaluating their potential in neural tissue engineering. J Biomater Sci Polym Ed. 2008;19(5):623-34.

74. Huang ZH, Wang Y, Cao L, Su ZD, Zhu YL, Chen YZ, Yuan XB, He C. Migratory properties of cultured olfactory ensheathing cells by single-cell migration assay. Cell Res 2008;18:479-490.

75. Ekberg JAK, Amaya D, Mackay-Sim A, St. John JA. The migratory of olfactory ensheathing cells during development and regeneration. Neurosignals published ahead of print March 27, 2012, doi: 10.1159/000330895.

76. Ruitenberg MJ, Vukovic J, Sarich J, Busfield SJ, Plant GW. Olfactory ensheathing cells: characteristics, genetic engineering, and therapeutic potential. J Neurotrauma 2006;23:468-78

77. Chaikin PM, Lubensky TC. Principles of condensed matter physics. Cambridge University Press, Cambridge, UK, 1995. Page 371

78. Simpon ML, KA; Hughes, BD. Cell invasion with proliferation mechanisms motivated by time-lapse data. Physica A. 2010;389:3779-90. 